\title{
CFD Simulation of Liquid-solid Multiphase Flow in Mud Mixer
}

\author{
T.Y. Kim ${ }^{\# 1}$, G.M. Jeon ${ }^{\# 2}$, J.C. Park ${ }^{\# 3}$, S.K. Sung ${ }^{* 3}$ and P.G. Kim ${ }^{* 4}$ \\ \# Department of Naval Architecture and Ocean Engineering, Pusan National University, Busan, South Korea \\ E-mail: ${ }^{1}$ taeyoung1012@pnu.edu; ${ }^{2}$ comjys0675@pnu.edu; ${ }^{3}$ jcpark@ pnu.edu \\ * Daewoo Shipbuilding \& Marine Engineering CO., LTD. Seoul, South Korea \\ E-mail: ${ }^{3}$ isac99@DSME.co.kr; ${ }^{4}$ pgkim1@DSME.co.kr
}

\begin{abstract}
In the present study, a computational fluid dynamics (CFD) simulation was performed to analyze the mixing phenomena associated with multi-phase flow in a mud mixing system. For the validation of CFD simulation, firstly a liquid-solid multiphase flow inside horizontal pipe was simulated and compared with the experiments and other numerical simulations. And then, the multiphase flow simulation was carried out for the mud mixer in the drilling handling system in order to understand mixing phenomena and predict the mixing efficiency. For the modeling and simulation, a commercial software, STAR-CCM+, based on a finite-volume method (FVM) was adopted. The simulation results for liquid-solid flow inside the pipe shows a good agreement with the experimental data. With the same multiphase model, the simulation for mud mixer is performed under the generalized boundary condition and then pressure drop through the mud mixer will be discussed.
\end{abstract}

Keywords - Mud handling system; Mud mixer; Liquid-solid multiphase flow; CFD; Mixing flow

\section{INTRODUCTION}

As well known, offshore oil drilling is a mechanical process using drilling mud to extract petroleum or natural gas through wellbore in seabed. Drilling mud, which is the mixture of oil (or water) and bulk (Barite, Bentonite, Polymer etc.), is of high viscosity and used to remove cuttings, provides hydrostatic pressure, cool down drilling bit and lubricate drilling bit. Although there are many different types of facilities from which offshore drilling operations take place, the core system of them is drilling system, which is mainly composed of mud-handling and bulk-handling systems.

When drilling operation is being performed, many physical and chemical changes are occurred in wellbore. To handle many changes of well condition and keep drilling process, additives of bulk are added into drilling mud through mud mixing system, such as bentonite for increasing density of drilling mud, barite for increasing viscosity of drilling mud, polymer for chemical control, or surfactant. Because the achievement of the required material property through mud mixing system is essential to stabilize drilling system, it is of importance to analyze multi-phase flow during mud mixing process, which is directly related to increase mixing performance of the system and guarantee the safety of the whole drilling system.
In this study, a computational fluid dynamics (CFD) simulation is applied to analyze the multi-phase flow and understand flow characteristics in mud mixer. Firstly, the liquid-solid multiphase flow in a horizontal pipe is simulated and compared with experimental results performed by [1] and other numerical results performed by [2],[3]. After then, multiphase simulation for generalized mud mixer model is also performed. For the present numerical simulation, commercial software STAR CCM+ based on the finite volume method (FVM) is used.

\section{GOVERning EQUATIONS AND NUMERICAL MODELING}

\section{A. Governing equations}

The governing equations for incompressible and viscous flow are the continuity and RaNS (Reynolds-averaged Navier-Stokes) equations as follows:

$$
\nabla \cdot \alpha_{i} \mathbf{u}_{i}=0
$$

$\frac{\partial \alpha_{i} \mathbf{u}_{i}}{\partial t}+\nabla \cdot \alpha_{i} \mathbf{u}_{i} \mathbf{u}_{i}=-\frac{1}{\rho_{i}} \nabla p+\nabla \cdot\left[\alpha_{i}\left(\tau_{i}+\tau_{i}^{t}\right)\right]+M_{i}+\left(F_{\mathrm{int}}\right)_{i}$

where, $\alpha_{i}$ is the volume fraction, $\mathbf{u}_{i}$ the velocity vector, $\tau_{i}$ stress tensor, $\tau_{i}^{t}$ the turbulent stress tensor, $\rho_{i}$ density, $p$ 
pressure, $M_{i}$ momentum transfer term and $F_{\text {int }}$ the internal forces.

\section{B. Numerical modeling}

In STAR-CCM+, Hybrid Gauss-LSQ method is adopted for pressure gradient term and 2nd-order upwind scheme for convection term. For solving the turbulent stress in governing equations, k-epsilon standard model is used. Drag, turbulent dispersion and lift forces model between each phase are adopted for momentum transfer. Internal force includes the pressure of solid that occurs only in the particle phase.

In this simulation, Syamlal O'Brien model [4] for drag force was adopted. The Syamlal O'Brien drag coefficient is based on measurements of terminal velocity in settling beds. The drag coefficient defines liquid-solid momentum exchanges as a function of the particle phase fraction, particle Reynolds number and terminal velocity. For predicting the distribution of solid particle in pipes, to predict the turbulent dispersion force is important [5]. To calculate turbulent dispersion, the turbulent Prandtl number 1 is adopted. For the lift force model, formula from [6] is used and the value of the lift coefficient $C_{L}$ is 0.1 , which is commonly used for small solid particles [5]. For more accurate representation of the solid particles than the simple solid pressure force model, the Granular flow model is adopted.

\section{NUMERCIAL SIMULATION}

\section{A. Validation for liquid-solid multiphase flow}

Liquid-solid multiphase flow in a horizontal pipe line is simulated and compared with experimental results [1] and other numerical results [2],[3]. In both experiment and CFD, length and diameter of horizontal line are around $10 \mathrm{~m}$ and $103 \mathrm{~mm}$, respectively, as shown in Fig. 1. The averaged velocity in inlet is $3 \mathrm{~m} / \mathrm{s}$ and concentration of solid particle is 0.19 . The material of phase- 1 is assumed as tap water and phase-2 as silica particles. The density and diameter of silica particle are set at $2650 \mathrm{~kg} / \mathrm{m}^{3}$ and $90 \mu \mathrm{m}$, respectively.

Firstly, to select the well-adapted drag model, two drag models, such as the Gidaspow model [7] and Syamlal O`Brien model [4], are tested through the comparative simulation, and the Syamlal O'Brien drag model [4] shows more reasonably-matched to the experiments than the other, as shown in Fig. 2. Hereby, the Syamlal O'Brien model [4] for drag force is employed to all of simulation.

The distribution of solid particles which is represented by volume fraction in horizontal pipe line is shown in Fig. 3. As solid particles are transported to the end of the pipe line, some of them sinks gradually below due to difference of density. Fig. 4 shows the magnitude of velocity in phase1(liquid), and the center of maximum value goes slightly up because the solid particles move downward.

The gird convergency are tested with $1,000,000$ to $5,000,000$ grid points, and the results are depicted in Fig. 5. It is seen that the solid concentration seems to be not so much affected by the number of grids.

In Fig. 6, the comparison of the present simulation is made with the experiment and other numerical simulations. As the results, it is seen that the present simulation represents very steep concentration profile of solid particles and is closer to the experiment than other numerical results.

\section{B. Simulation for mud mixer}

Next, the simulation of multiphase flow for mud mixer is performed to find the optimal configuration which can improve mixing performance.

The geometry of mud mixer is derived from the statistical data of manufactured products and schematic view of simply-designed mud mixer is shown in Fig.7. Here, the diameter of mud pipe line is $0.15 \mathrm{~m}$ and liquid capacity is 600 1200gpm.

Fig. 8 shows the change of pressure proved at the mud inlet along time. The maximum pressure appears around $17 \mathrm{~s}$, and a quasi-steady state is reached after 70 s. It means that the evaluation for mixing performance of mud mixer is available after 70s. After reaching at the quasi-steady state, the volume fraction of mud at the center of hopper is proved over 0.5 . It means that the mud is partially transported backward because of the small diameter of diffuser.

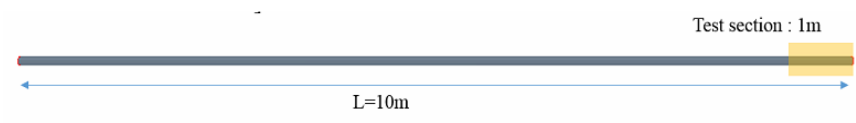

Fig.1 Geometry of horizontal pipe line

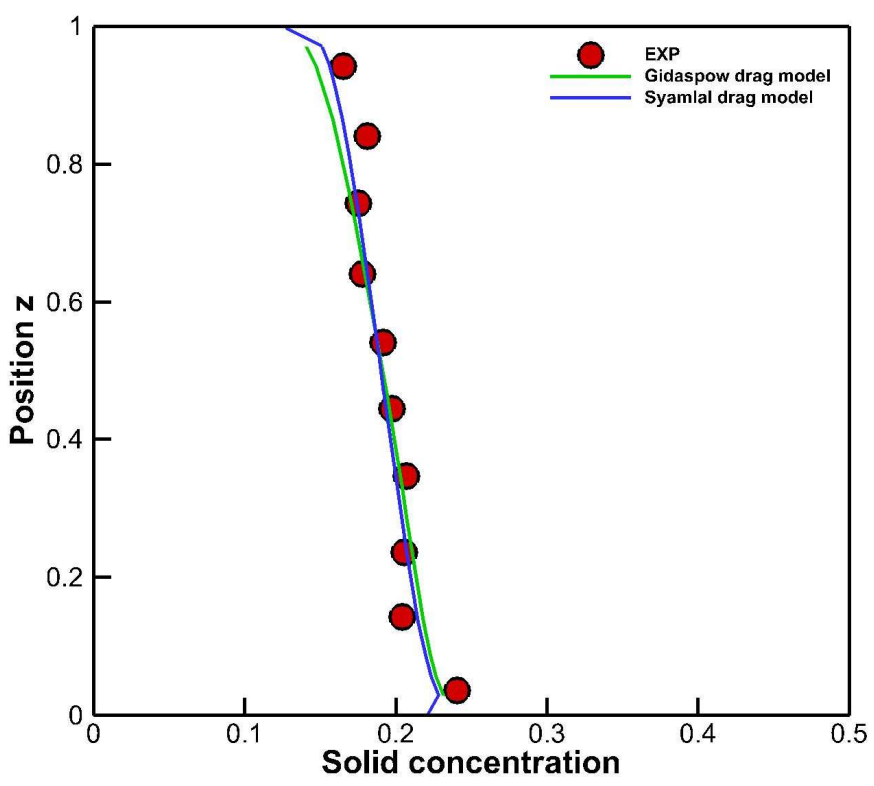

Fig.2 Comparison of solid concentration for drag model test
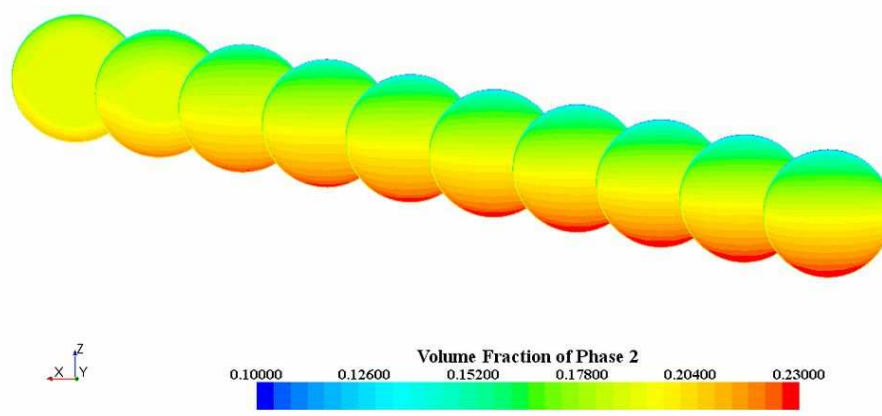

Fig.3 Volume fraction of phase-2 (Solid) 

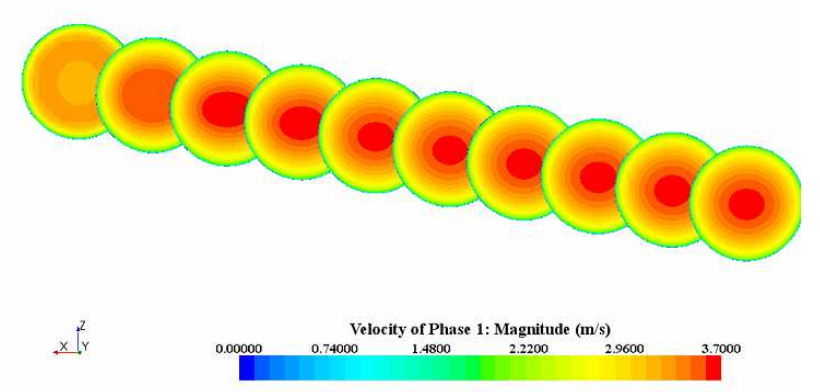

Fig.4 Velocity of phase-1(Liquid)

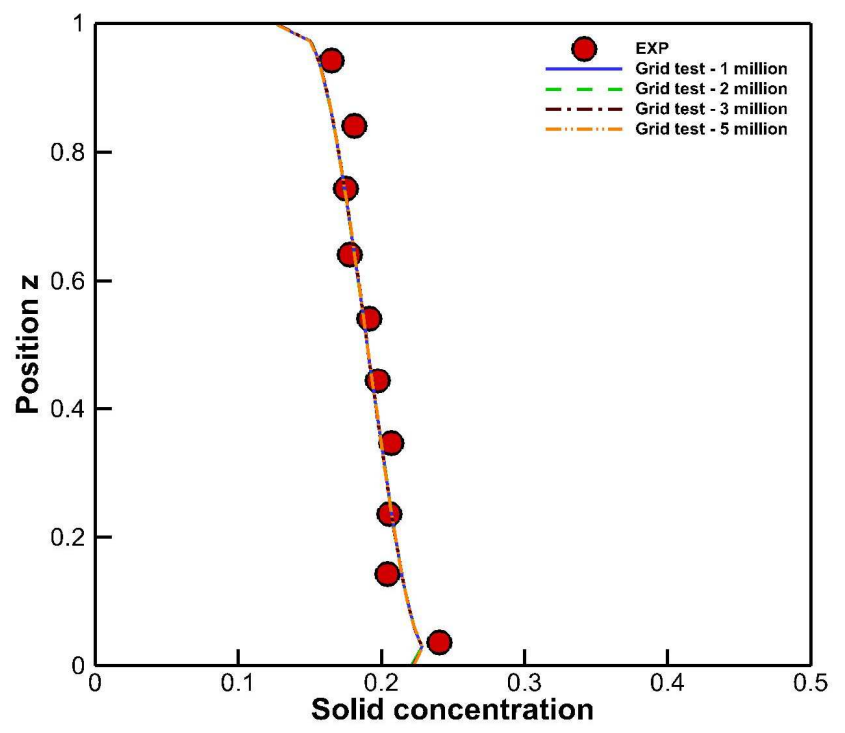

Fig.5 Grid convergence test

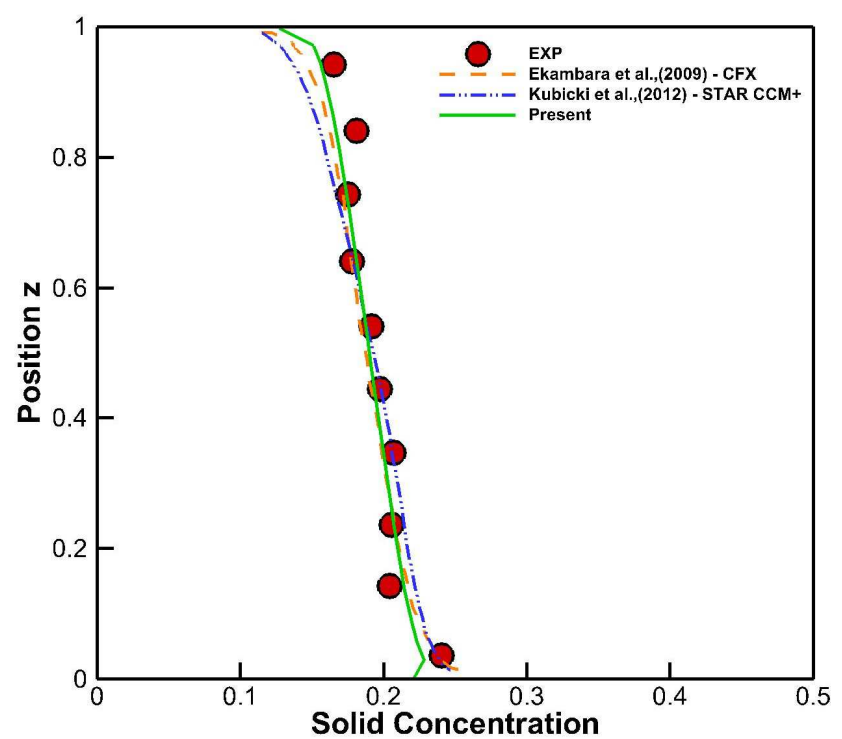

Fig.6 Comparison with experimental and numerical results

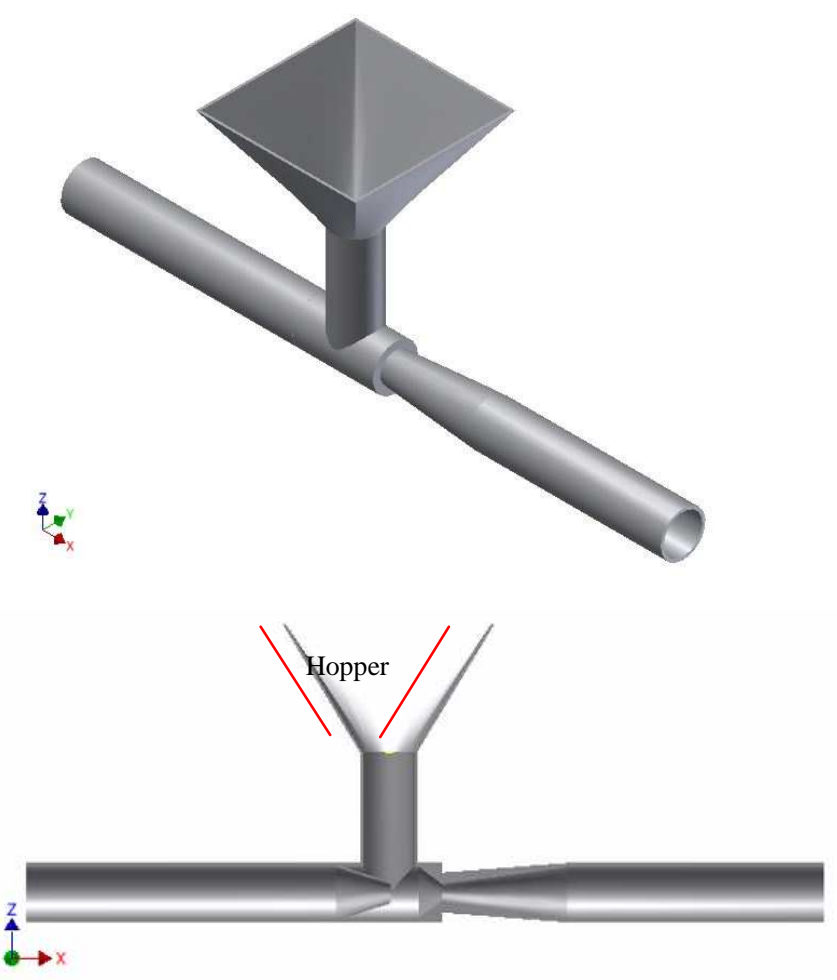

Fig.7 Schematic view of designed mud mixer

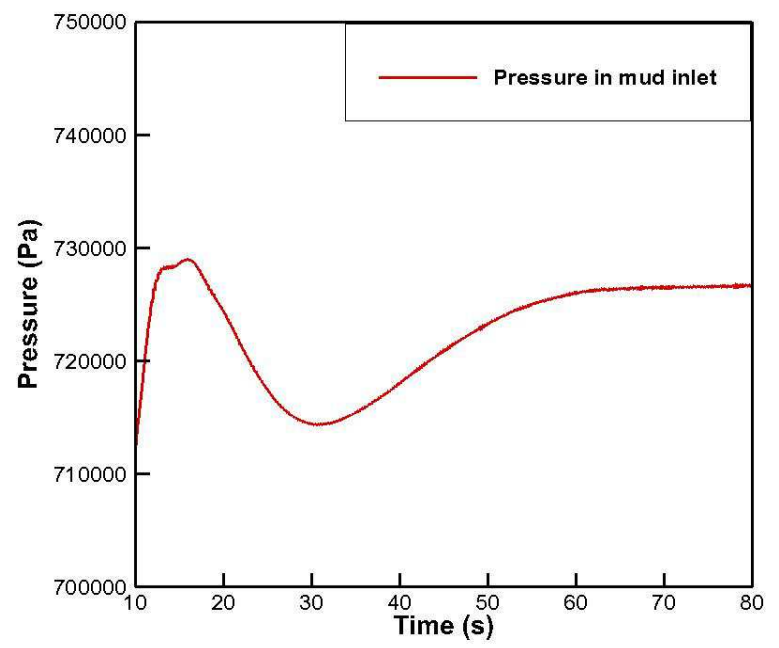

Fig.8 Change of pressure at mud inlet along time

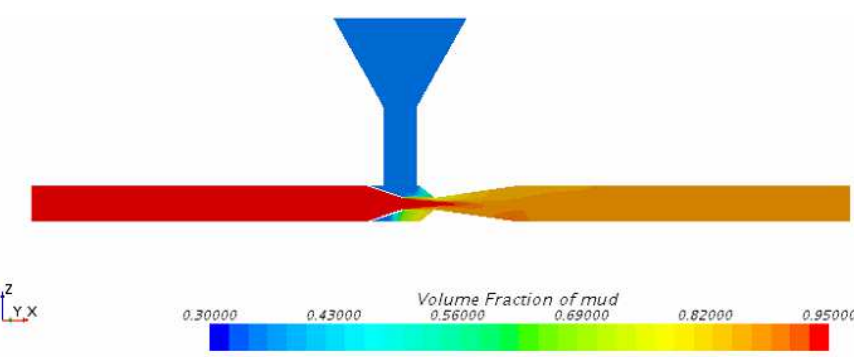

Fig.9 Volume fraction of mud at $80 \mathrm{~s}$ 


\section{CONCLUSIONS}

In this study, a computational fluid dynamics (CFD) simulation was applied to analyze the multi-phase flow and understand flow characteristics in mud mixer. The liquidsolid multiphase flow in a horizontal pipe was simulated to check the proper drag model and compared with experiments [1] and other numerical simulations [2],[3]. As the results, it seemed that the present simulation for solid concentration was agreed with the experiment compare to other numerical results when the Syamlal O`Brien`s model [4] was employed to liquid-solid multiphase flow in a pipe.

In the multiphase simulation for the mud mixer, the simply-designed mud mixer derived from the statistical data of manufactured products was introduced and liquid-solid flow in the mixer was simulated. It can be said that the CFD simulation indicates the potential for multiphase flow in mud mixer and is quite useful for M\&S (Modeling and simulation)-based design of optimized mixer in mud handling system.

\section{ACKNOWLEDGMENT}

This research was supported by the Industrial Strategic Technology Development Program (G01201306010380, Bulk and LP Mud Handling System for Deep Sea Drilling System) funded by the MOTIE/KEIT.

\section{REFERENCES}

[1] 1. R.G. Gillies, C.A. Shook and J. Xu, "Modeling heterogeneous slurry flows at high velocities,". Can. J. Chem. Eng., vol. 82, pp. 1060-1065, October 2004.

[2] D. Kubicki and S. Lo, "Slurry transport in a pipeline - Comparison of CFD and DEM models,". Ninth Internatonal Conference on CFD in the Minerals and Process Industries, December 2012.

[3] K. Ekambara, R.S. Sanders, K. Nandakumar and J.H. Masliyah, "Hydrodynamic simulation of horizontal slurry pipeline flow using ANSYS-CFX,". Ind. Eng. Chem. Res., vol. 48, pp. 8159-8171, May 2009.

[4] G. Kathuria, A. Karnik and M. Tandon, "Study of gas-solid fluidized bed - Tuning Syamlal O Brien drag model", in technical report from CD-adapco, DOI: 10.13140/RG.2.1.4300.5609/1, 2015

[5] T.Y Kim and J.C.Park, "CFD simulation of multi-phase mixing flow in mud mixing system," unpublished, 2015.

[6] T.R Auton., J.C.R. Hunt and M. Prud 'Homme, "The force exerted on a body in inviscid unsteady ono-uniform rotational flow,". J. Fluid Mech., vol.197, pp. 241-257,December 1988.

[7] D. Gidaspow, "Multiphase flow and fluidzation - Continum and kinetic theory description", in academic press, 1994 